\title{
Designing scheduling concept and computer support in the food process- ing industries
}

D.P.van Donk, W. van Wezel and G. Gaalman

Faculty of Management and Organisation, University of Groningen,

P.O. Box 800, 9700 AV Groningen, The Netherlands, Tel. +3150 3637345, fax. + 31503632032 ,

e-mail:D.P.VAN.DONK@BDK.RUG.NL.

\begin{abstract}
Food processing industries cope with a specific production process and a dynamic market. Scheduling the production process is thus important in being competitive. This paper proposes a hierarchical concept for structuring the scheduling and describes the (computer) support needed for this concept.
\end{abstract}

\section{Keywords}

Food processing industry, scheduling, hierarchy, computer support. 


\section{INTRODUCTION}

Nowadays an often asked question in food processing industries relates to improving production schedules. The background of this question seems in many cases twofold. On the one hand there are a number of industry specific characteristics, which cause complexity and uncertainty. On the other hand there is growing necessity to meet higher customer demand both in logistical requirements and in a need for introducing new products.

Complexity in production in food processing industry arises from the number of steps such as: receiving materials, mixing/blending according to recipe, forming and processing (e.g. sterilizing), consumer packaging and adding a case packing, storage until delivery to clients. Moreover, production planning and control in these industries has to cope with specific characteristics. These include high capital intensity in the process stage and high labour intensity in the packaging stage; limited storage-live for both the raw materials and the end-products; hygienic factors causing considerable (often sequence-dependent) set-up times; flow-like production with limited work-in-progress; variability in yields as well as in the quality of raw materials. Balancing different capacities and different stages in the production can be a problem as well.

Market requirements tend to increase the number of (new) end-products in a large variety of different packages (sizes and brands). Complexity in scheduling is further increased by a growing number of recipes (each having a specific need of machine capacity and labour requirements), a growing percentage of make-toorder mixed with the traditional make-to-stock. Furthermore, there is an emphasis on delivery speed, dependability and traceability and, simultaneously, a tendency to reduce stocks.

As a result, scheduling in the food processing industry can be characterised as a vital function in which on the one hand market needs have to be met and on the other hand, internal performance measures. That means balancing costs (by efficient use of available capacity and low inventories), quality, delivery times and still being flexible (cf. Jakeman, 1994; Nakhla, 1995). The necessity emerges to support scheduling in food processing. In the words of Jakeman: "Without computer-aided scheduling tools production staff cannot see the full consequences of their actions". However, previous research (Van Dam et al., 1993) showed that most detailed planning and scheduling is still performed manually. This is even more striking as there are a large number of software packages aimed at supporting scheduling, available (Benoy et al., 1994). Implementation of these packages seems to be lacking behind. The underlying reasons might be the high price of scheduling systems, the alignment to the task of the scheduler or the adjustment to the specific characteristics of the organisation (Van Wezel and Van Donk, 1996).

The objective of this paper is to investigate how we can support scheduling in 
food processing industries. A constraint of this paper might be that the food processing industry in the Netherlands is a sector with relatively many small and medium-sized companies with 60-100 people employed. Our observations and recommendations might be restricted to that class of organisations.

Based on observations made in previous work a hierarchical concept for scheduling will be developed. Implementation of such a concept is enabled through computer support. Underlying the computer support is the important observation that scheduling decisions are taken within an organisational context. This will elaborated upon. The last section will give some conclusions and discussion for further research.

\section{DESIGNING A SCHEDULING HIERARCHY}

Before actually designing a scheduling concept we like to emphasize the main reasons underlying the design. First of all, scheduling in the food processing industry has in most cases to cope with a number of interrelated scheduling problems (see Van Dam et al., 1993). The task of a scheduler can be broken down into several elementary scheduling problems (such as sequence dependent set-ups, shared resources, similar machines) which are frequently studied in the literature as (single) problem, which are strongly related in practice. Secondly, there is a number of uncertainties regarding market demands, production capabilities and capacities. Moreover, all kind of irregularities might happen during the execution of the schedule: rush orders, machine break-downs etc. Thirdly, before actually scheduling lots of information has to be gathered, stored, interpreted and communicated.

In designing a scheduling concept we choose to distinguish two interrelated parts: the design of the decision processes and the design of the information support system. In the design of the decision processes we will follow some previous work in which a hierarchy is proposed. The information support system is adapted to this hierarchy and will be described in the third section of this paper.

\subsection{A starting point: two levels of scheduling}

The basis of the design of a (decision-)hierarchy for scheduling lies in the analyses of the structure of the scheduling situation, and subsequently, the distinction of the various scheduling characteristics in that situation, and related scheduling decisions. The previous sections unveiled the complexity in scheduling and the complexity for decision-making in scheduling. In such situations a hierarchical approach has the advantage that the complexity is reduced on each separate level. Decomposition of the total problem is therefore critical for the success of such an approach. Too much or too little decisions on one level makes the decomposition meaningless (Mesarovic et al., 1970). A hierarchical approach is understood as an 
approach in which the total amount of decisions is divided among several decisions-levels, in such a way that a higher level gives instructions, constraints and conditions for a lower level to solve the lower level's problems (Mesarovic et al., 1970). Each level solves it's own problems and feedback is given to the next higher level.

Van Dam et al. (1993) show that short term scheduling decisions in practice are usually made at two levels: a higher level labelled the Detailed Planning Level and a lower level labelled the Scheduling Level. The Detailed Planning Level deals with the establishment of prerequisites that extend over individual orders. Decisions at the Detailed Planning Level are made on the basis of more aggregate information and measures. Typical decisions include balancing of capacities (decisions with regard to overtime, number of shifts, use of production lines, number of employees, stock replenishment, etc.), purchasing of (packaging) materials, and, sometimes, the sequence of production. The typical horizon of the Detailed Planning Level is two weeks up to several months and the planning periods are weeks or months. The Scheduling Level deals in the opinion of Van Dam et al. (1993) with sequencing, the determination of starting and finishing times, the allocation of personnel to (packaging) lines, and rescheduling. Usually on this level the planning horizon is a week up to a month (sometimes a day) and the planning periods are hours, days and sometimes weeks.

The Detailed Planning Level sets the boundaries for the Scheduling Level. In other words the Scheduling Level decides on the basis of the decisions taken at the Detailed Planning Level. The Detailed Planning Level on the other hand decides among others, on the basis of the actual performance (feedback) of the Scheduling Level. The Scheduling Level itself receives feedback from the production process: e.g. deviations from the planning and unanticipated events.

\subsection{The hierarchy in practice}

As we all know a schedule will usually not be realized as it is dispatched. A number of reasons exists: the schedule has political (e.g. the schedule is too optimistic) statements in it (McKay et al., 1995), capacities of machines are higher/lower then planned for, raw materials are not available due too quality problems, an extra order arrives for an important customer. Although everybody seems to know that schedules will not be realized as initially intended, no explicit and organised actions are undertaken to deal with the deviations.

We observed that the hierarchy more or less works in one way: from the higher level(s) down to the lower level(s) and the shopfloor without formal organised feedback. Mesarovic et al. argue that feedback is important in hierarchical systems to have a properly functioning system. In other words, organisational arrangements and procedures lack to adapt schedules rapidly if the situation changes. In fact there is usually not an organised way to communicate changes in the 
schedule to the shopfloor. Also adaption of the plan from the Detailed Planning Level is not easy as little feedback is given to that Level. Most schedulers lack instruments to evaluate alternative schedules or the consequences of adapting a schedule. As a result, improvements and learning in scheduling is not supported and quick responses to changes are difficult made.

\subsection{A hierarchy of scheduling decisions}

Considering the observations in the previous section, our initial hierarchy needs to be worked out more explicitly. First of all, feedback needs more emphasize in the hierarchy, in line with the ideas of Mesarovic et al. (1970). Feedback from the Scheduling Level helps the Detailed Planning Level in learning the uncertainties on the lower level and thus better aggregate plans can be made. Secondly, we propose to distinguish Rescheduling as a separate part of the scheduling concept to deal with the above mentioned characteristics and especially the uncertainty of the production process and the flexibility needed from the market. Here as well, feedback from the production process to the Scheduling Level is made explicit, which enables better plans.

Figure 1 shows the relations between the parts of the Scheduling Concept.

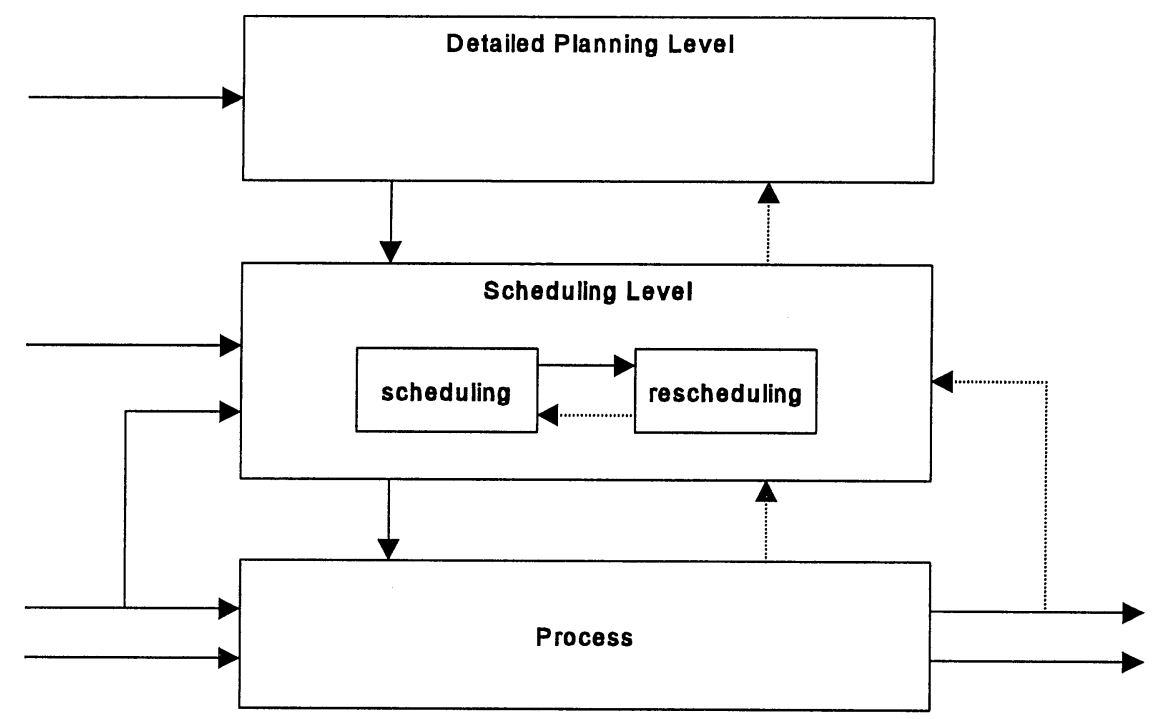

Figure 1 A conceptual model of the scheduling hierarchy.

At the Detailed Planning Level (DPL) information is needed regarding large orders from (foreign) customers, stock levels, forecasts, desired production efficiency, due dates, etc. The information is used to build an aggregate plan 
which balances capacity and assigns orders to periods. Decisions on this level are taken under uncertainty but can not be postponed until more accurate information is available. The plan is put downward to the Scheduling Level (SL). A the DPL feedback, regarding past performance and uncertainties, is taken into account (the dotted arrow from SL to DPL). The SL builds its schedule using the plan from the DPL. Information regarding customers and stock orders are needed as well as information on recent performance and peculiarities of the production process and the inputs for the production process (e.g. stock levels of raw materials. The scheduling part of the SL dispatches a schedule to the shopfloor or feedback is given to the DPL if inconsistencies are detected. During the execution of the schedule all kind of events may happen related to customers, the process or the output of the production process. This is dealt with in the Rescheduling part of the SL and results in an adaption to the initial schedule (and subsequent communication to the process). To perform the rescheduling the initial plan is available for the Rescheduling part and feedback is given to the Scheduling Part to enhance learning and enable the scheduling part in making better forecasts e.g. related to the performance of a machine. Feedback is also given if the schedule is executed according to plan. Feedback from the SL to the DPL yields better insight for the DPL in the uncertainties on the SL. Ultimately, adaption of the schedule at the DPL may be needed. Part of the information flow through the process is not visible as is depicted at the bottom of Figure 1.

Good functioning of the Rescheduling System and the other parts of the concept is facilitated by adequate computer support. We will pay attention to computer support in the next section.

\section{COMPUTER SUPPORT}

The work of scheduling is more than just solving the problem of finding suitable start and finish times for orders. Much effort of the planner is put in finding and (re)arranging information, checking constraints, interpreting information and negotiating plans and make adaptions to schedules. It might be obvious that scheduling support by computers can be advantageous in structured tasks like counting, rearranging of numbers or performing computations. Human beings are better in qualitative reasoning and the use of vague information. Cognitive sciences can help in the division of tasks and decisions between the scheduler and the computer (e.g. Prietula, 1994). This section will particularly pay attention to the (computer) support needed in implementing the hierarchy. Pivotal in that is the working and the support of Rescheduling.

Computer support of rescheduling is first of all supporting the availability of information regarding machine and material performance, new orders, unexpected events, etc. That seems obvious, but is still far away from current practice. 
Most companies collect customers order and hand these over to the scheduler once a week and thus miss opportunities to produce an order earlier or combine different orders in one production batch.

A second point of concern is the relation between Scheduling and Rescheduling. Scheduling is making a schedule in a constructive way, while Rescheduling schedules in a reactive way. Constructive scheduling may need the availability of optimising tools and algorithms, while reactive scheduling adapts an existing plan. In adapting an existing schedule the main goal is to rearrange production orders, while not violating constraints and changing the schedule as little as possible. Computer support can be helpful in suggesting alternatives and showing their consequences or checking constraints (capacity, availability of materials). Most existing scheduling systems mainly support constructive scheduling, while the facilities for manipulation are limited. When decisions are computer supported, communication down to the shop floor should be better organized as well. A third point to look at is the relation between the Detailed Planning Level and Rescheduling. Traditionally it is quite common to have feedback from a lower level to a higher level within time frames of a week or month. Whenever the scheduler is confronted with a problem he can not solve at the Scheduling Level it should be made possible to look at the Detailed Planning Level. A number of options is possible. It might be that rearranging orders from one period to an other is possible within the constraints of the Detailed Planning Level. Also the possibility of postponing an order to another period needs to be investigated. More generally, the computer support should be supporting the communication between the planning levels and support easy exchange between these levels.

\section{CONCLUSION AND FURTHER RESEARCH}

Food processing industries have some special characteristics and operate in a market with high logistical demands. Scheduling is therefore important in being competitive. Based on earlier observations a hierarchical concept for scheduling is elaborated and the computer support needed, is described.

Of course some questions need to be answered. Further elaboration of the relations between the DPL, SL and Rescheduling is needed, as well as the level of aggregation and detail needed on each level. We also need measures to adapt the general frame to specific situations. To find answers to these questions some case-studies are currently undertaken, in which computer support of the hierarchical concept but also for other tasks of the scheduler is designed. 


\section{REFERENCES}

Benoy, M. , DeWilde, P., Voet, M. and Herroelen, W. (1994) Finite Scheduling: State-of-the-Market, Ernst \& Yound Management Consultants, Brussel.

Jakeman, C.M. (1994) Scheduling needs of the food processing industry, in Food Research International, 27, 117-120.

McKay, K.M., Safayeni, F.R. and Buzacott, J.A. (1995) 'Common sense' realities of planning and scheduling in printed circuit board production, in International Journal of Production Research, 33, 1587-1603.

Mesarovic, M., Macko, D. and Takahara, Y. (1970) Theory of Hierarchical, Multilevel Systems, Academic Press Inc., New York/London.

Nakhla, M. (1995) Production control in the food processing industry: the need for flexibility in operations scheduling, in International Journal of Operations \& Production Management, 15, 73-88.

Prietula, M.J., Wen-Ling Hsu, Peng Si Ow, and Thompson, G.L. (1994) MACMERL: Mixed-initiative scheduling with coincident problem space, in Intelligent Scheduling (Ed. M. Zweben and M.S. Fox), Morgan Kaufman Publishers, San Francisco.

Van Dam, J.P., Gaalman, G.J.C. and Sierksma, G. (1993) Scheduling of packaging lines in the process industry: An empirical investigation, in International Journal of Production Economics, 30-31, 579-589.

Van Wezel, W. and Van Donk, D.P. (1996) Scheduling in food processing industries: preliminary findings of a task oriented approach, in Proceedings second international conference on Computer Integrated manufacturing in the Process Industries (Ed. J.C. Fransoo and W.G.M.M. Rutten), BETA, Eindhoven, 545-557. 